\title{
Bladder Perforation of a Ventriculoperitoneal Shunt Catheter: Case Report and Literature Review
}

\author{
Gema Romeu Magraner¹, Eduardo Morán Pascual*1, Laura Barrios Arnau², Arnold R Quiroz Tejada ${ }^{3}$, Sara Villarroya \\ Castillo $^{1}$ and Enrique Broseta Rico ${ }^{1}$
}

${ }^{1}$ Department of Urology, Hospital Universitario y Politécnico La Fe, Spain

${ }^{2}$ Department of Urology, Hospital General Universitario de Castellón, Spain

${ }^{3}$ Department of Neurosurgery, Hospital Universitario y Politécnico La Fe, Spain

Received: 眥: October 10, 2018; Published: 輩: October 24, 2018

*Corresponding author: Eduardo Moran Pascual, Department of Urology, Hospital Universitario y Politécnico La Fe, Avda. Fernando Abril Martorell, 106, Valencia, 46026, Comunidad Valenciana, Spain

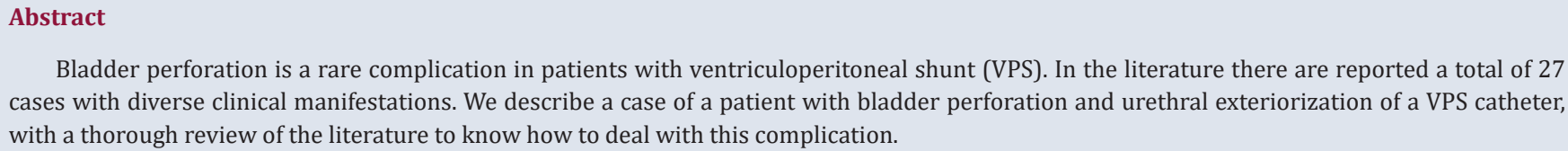

Bladder perforation is a rare complication in patients with ventriculoperitoneal shunt (VPS). In the literature there are reported a total of 27 cases with diverse clinical manifestations. We describe a case of a patient with bladder perforation and urethral exteriorization of a VPS catheter, with a thorough review of the literature to know how to deal with this complication.

Keywords: Bladder perforation; Urethra; Complication; Ventriculoperitoneal shunt

\section{Introduction}

The placement of a VPS catheter is the most frequent procedure for the treatment of hydrocephalus. Perforation of abdominal viscera of the distal end of the catheter are rare complications described in the literature. The first cases of spontaneous bladder perforation were reported by Grosfeld et al. [1] in 1974, who described two cases in two pediatric patients. Since then, a total of 27 cases have been published. This complication can have different clinical manifestations. Being a rare pathology, there is no consensus on the treatment, however, most authors recommend replacing the system and antimicrobial therapy [2]. We describe another the case and a review of the literature.

\section{Clinical Case}

This is a 23-year-old woman with a history of cranioencephalic trauma when she was one year old and therefore epilepsy and noncommunicating hydrocephalus as consequence, a VPS catheter was placed. At the age of 12 a replacement of the bypass system due to malfunctioning was required. She presented normal controls until she was 16 years old. After 7 years without follow-up, she went to the emergency room with a tonic-clonic seizure with associated incontinence that was initially correlated with a poor adherence to antiepileptic medication. Because she also presented discomfort in the VPS catheter pathway, a magnetic resonance, plain radiograph and abdominal ultrasound were requested. Imaging showed an intraperitoneal path of the catheter surrounded by fat with inflammatory chances and free peritoneal fluid. During the study she complained about an intermittent VPS catheter exteriorization through urethra during micturition. Physical examination confirmed this fact.

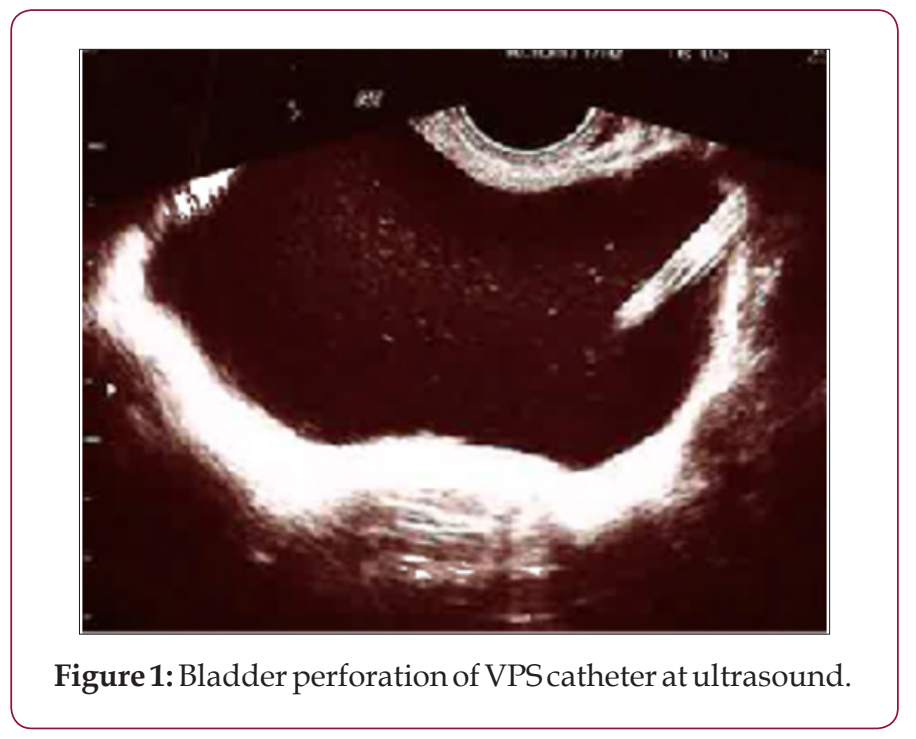

Ultrasound and abdominal radiography corroborated the presence of the intravesical catheter (Figure 1). Given the results, an urgent surgery was scheduled together with the neurosurgeon, 
where an abdominal incision was made with VPS catheter exteriorization and resection of its distal end. We didn't repair the bladder wall. In addition, samples from the cerebrospinal fluid (CSF) and the distal end of the catheter were sent for culture. Foley catheter was placed, and empirical antimicrobial therapy was started. During admission, the rest of the VPS system was eventually removed due to a positive result of the culture of cerebrospinal fluid for Staphylococcus capitis; antimicrobial therapy was adjusted to the antibiogram and was maintained for 21 days. The patient was discharged with a Foley catheter. After 15 days, a cystography ruled out any urinary fistula, so the Foley catheter was removed. Currently, the patient remains without a bypass system and has not presented any complications.

\section{Discussion}

Abdominal viscera perforation or distal end extrusion of the catheter are uncommon complications of VPS. This complication is much more frequent in the pediatric age (67.9\%) and there seems to be no gender association or any link with the etiology of hydrocephalus (Table 1). Some authors argue that bladder perforation may be related to a bigger bladder volume [2] as they observed this complication in 8 patients with an augmented bladder (28.6\%). Regarding the clinical presentation, 20 patients (71.4\%) presented catheter exteriorization through the urethra, approximately half of them being asymptomatic, as occurred in our case. Ten patients (35.7\%) presented with urinary symptoms, $7(25 \%)$ abdominal pain and $3(10.7 \%)$ with infection signs. In general, urethral exteriorization is easy to diagnose, but in some patients, symptoms can be variable and make clinical suspicion difficult. Even calcification of the catheter [3-6] has been described, but this usually requires a long exposure time. On the other hand, lower urinary tract symptoms (LUTS) may occur in the absence of infection.

Table 1: Clinical and demographic characteristics.

\begin{tabular}{|c|c|c|}
\hline Characteristics & Number of cases (n=28) & Percentage \\
\hline Age & 19 & $67.9 \%$ \\
$>18$ & 6 & $21.4 \%$ \\
Missing data & 3 & $10.7 \%$ \\
\hline Gender Female & 12 & $42.9 \%$ \\
Male & 10 & $35.7 \%$ \\
Missing data & 6 & $21.4 \%$ \\
\hline Type of bladder Normal & 20 & $71.4 \%$ \\
Augmented & 8 & $28.6 \%$ \\
Missing data & 10 & $0 \%$ \\
\hline Hydrocephalus Etiology & 7 & $35.7 \% 25 \%$ \\
Spina bifida & 4 & $14.3 \%$ \\
Congenital & 3 & $10.7 \%$ \\
Post hemorrhage & 4 & \\
Tumor related & & \\
Others & & \\
\hline
\end{tabular}

\begin{tabular}{|c|c|c|}
\hline $\begin{array}{l}\text { Number of shunt } \\
\text { revisions }\end{array}$ & 7 & $25 \%$ \\
\hline Without revisions & 6 & $21.4 \%$ \\
\hline 1 revision & 4 & $14.3 \%$ \\
\hline Multiple & 11 & $39.3 \%$ \\
\hline \multicolumn{3}{|l|}{ Missing data } \\
\hline \\
\hline <= 1 year & & \\
\hline 1-5 years & 8 & $28.6 \%$ \\
\hline$>5$ years & 6 & $21.4 \%$ \\
\hline \multicolumn{3}{|l|}{ Missing data } \\
\hline $\begin{array}{l}\text { Clinical presentation } \\
\text { Catheter extrusion }\end{array}$ & 20 & $71.4 \%$ \\
\hline Asymptomatic & 9 & $45 \%$ \\
\hline With other symptoms & 11 & $55 \%$ \\
\hline Urinary symptoms & 10 & $35.7 \%$ \\
\hline ITU & 5 & $50 \%$ \\
\hline STUI & 5 & $50 \%$ \\
\hline Perforation Suspect & 2 & $20 \%$ \\
\hline Abdominal pain & 7 & $25 \%$ \\
\hline Catheter infection & 3 & $10.7 \%$ \\
\hline Meningitis & 2 & $66.7 \%$ \\
\hline Abdominal abscess & 1 & $33.3 \%$ \\
\hline Shunt status & 25 & 8930 \\
\hline Normofunctioning & 1 & $3.6 \%$ \\
\hline Non-functioning & 2 & $7.1 \%$ \\
\hline Missing data & & \\
\hline
\end{tabular}

So, this complication should be considered in this patient. Regarding diagnosis, it is recommended at least to perform an abdominal ultrasound [2] as we did in our case. Prasad et al. [7] suggested that this perforation is caused by a series of events such as catheter fixation, penetration and eventual perforation of the bladder wall, with subsequent exteriorization through the urethra [7,8]. De Aquino et al. [9] argue that the presence of inflammatory phenomena can facilitate the adhesion of the catheter to a viscera, which together with the increase of intra-abdominal pressure and peristaltic movements can produce the erosion and perforation $[8,9]$. This could explain what occurred in our case since days before we had detected the presence of free intraperitoneal fluid and fat with inflammatory characteristics around the catheter. The most life-threatening complication is infection of the system, with urinary tract infection being the most frequent cause in 5 patients $[5,6,10-12]$ followed by meningitis in 2 patients $[13,14]$. In these latter cases, the patients presented with neurological signs and symptoms and systemic infection.

In our case, the patient did not present signs or symptoms of infection initially, but the culture of the CSF was positive. For this reason, we recommend the culture of both, the CSF and the removed system, as well as other authors do [8]. Some authors recommend 
two treatment strategies [2]. In case of externalization of the catheter through the urethra, a suprapubic medial laparotomy is recommended to access the abdominal cavity, cut the catheter near to the bladder wall, repair the bladder wall and relocate the intraperitoneal end. In case of non-exteriorization to the outside, should to conduct a cystoscopy, cut the catheter as close as possible to the bladder wall and extract the distal end. In both cases, the placement of a bladder catheter is suggested. When it comes to the VPS handling, most authors recommend the withdrawal of the entire system $(42.9 \%)$ or the removal of the distal end with external drainage of the rest of the system (25\%). In our case, finally we decided to remove the complete system. In all cases it is recommended to initiate antimicrobial therapy in case of signs of infection, and adjust the duration and spectrum depending on the suspicious focus (urinary, meningeal or abdominal).

In case of absence of signs of infection, it is recommended the use of a broad-spectrum antibiotic therapy in a single prophylactic dose as in any other surgery. In 12 cases (42.9\%) laparotomy was decided, as in our case, and in 6 (21.4\%) diagnostic cystoscopy was the only procedure (Table 2). The repair of the defect in the bladder wall was only needed in 10 cases (35\%), the rest opted for conservative procedure placing a urethral catheter as we did in our case. Urinary fistula or urine leakage in the peritoneal cavity are not reported in the literature. In 16 patients (57.1\%) bladder catheterization was performed, in the majority of cases it was maintained for 5 to 7 days $(43.8 \%)$. In our case the catheter was in place for 2 weeks. We also conducted a cystography prior to its removal to ensure the complete closure of the bladder defect. Regarding the subsequent evolution, no long-term complications were observed except in one patient who died on the eleventh postoperative day [15-22] due to an electrolyte imbalance secondary to a tubular renal acidosis. In our case, we had no complications.

Table 2: Management and Evolution.

\begin{tabular}{|c|c|c|}
\hline Treatment & Number of cases (n=28) & Percentage \\
\hline Focus & 12 & $42.9 \%$ \\
Laparotomy & 6 & $21.4 \%$ \\
Cystoscopy & 3 & $10.7 \%$ \\
None & 7 & $25 \%$ \\
Missing data & 10 & \\
\hline Bladder repair & 14 & $14.7 \% 50 \%$ \\
Yes & 4 & \\
No & 16 & $57.1 \%$ \\
Missing data & 12 & $42.9 \%$ \\
\hline Foley / cystostomy Yes & 4 & $25 \%$ \\
Missing data & 3 & $18.8 \%$ \\
\hline Catheter time & 3 & $37.5 \%$ \\
5 days & 6 & \\
7 days & & \\
\hline 15 days & & \\
Missing data & & \\
\hline
\end{tabular}

\begin{tabular}{|c|c|c|}
\hline Shunt handling & 12 & $42.9 \%$ \\
Removal & 7 & $25 \%$ \\
External drainage & 2 & $7.1 \%$ \\
Review & 2 & $7.1 \%$ \\
Extruded end removal & 3 & $10.7 \%$ \\
Placement & 2 & $7.1 \%$ \\
Missing data & 16 & $57.1 \%$ \\
\hline Antibiotic Yes & 2 & $7.1 \%$ \\
\hline 1 day & 2 & $7.1 \%$ \\
\hline 2-7 days & 6 & $21.4 \%$ \\
8-14 days & 6 & $21.4 \%$ \\
Unknown & 12 & $42.9 \%$ \\
\hline Missing data & & \\
\hline
\end{tabular}

\section{Conclusion}

Bladder perforation is a rare complication of VPS catheters. In cases where transurethral exteriorization occurs the diagnosis is simple, for the rest, it is recommended to perform at least an imaging test such as ultrasound. Treatment is controversial given the low frequency of this pathology, but most authors choose to perform a laparotomy removing the complete device or external drainage [22-25]. Bladder catheterization for a minimum of 5 days and a broad-spectrum antimicrobial therapy is recommended.

\section{Acknowledgement}

We acknowledge Marta Romeu Pérez for participating in the technical editing and translation of the manuscript. The authors have no ethical conflicts to disclose, conflicts of interest to declare or any financial support.

\section{References}

1. Grosfeld JL, Cooney DR, Smith J, Campbell RL (1974) Intra-abdominal complications following ventriculoperitoneal shunt procedures. Pediatrics 54(6): 791-796.

2. Miranda ME, de Sousa MB, Tatsuo ES, Quites LV, Giannetti AV (2016) Bladder perforation by ventriculoperitoneal shunt. Child's Nerv Syst 32(12): 2321-2326

3. Eichel L, Allende R, Mevorach RA, Hulbert WC, Rabinowitz R (2002) Bladder calculus formation and urinary retention secondary to perforation of a normal bladder by a ventriculoperitoneal shunt. Urology 60(2): 344 .

4. Ramana Murthy KV, Jayaram Reddy S, Prasad DVSRK (2009) Perforation of the distal end of the ventriculoperitoneal shunt into the bladder with calculus formation. Pediatr Neurosurg 45(1): 53-55.

5. Butler L, Keys C LJ (2013) Bladder calculus for- mation on the tip of a migrated disused ven- triculoperitoneal shunt. J Pediatr Surg 48: E1-E3.

6. Ibrahim AK (2014) Urinary bladder stone complicating ventriculovesical shunt. Sultan Qaboos Univ Med J 14: e142-144.

7. Prasad VSS V, Krishna AM, Gupta PK (1995) Extrusion of peritoneal catheter of ventriculoperitoneal shunt through the urethra. Br J Neurosurg 9(2): 209-210.

8. Mutlu M, Kader Ş, Aslan Y, Yazar U, İmamoğlu M (2015) An Acute Complication of Ventriculoperitoneal Shunt with Bladder Perforation and Extrusion through the Urethra in a Newborn: Case Report and Review of the Literature. Pediatr Neurosurg 50(5): 264-269. 
9. De Aquino HB, Carelli EF, Borges Neto AG PC (2006) Nonfunctional abdominal complications of the distal catheter on the treatment of hydrocephalus: an inflammatory hypothesis? Experience with six cases. Childs Nerv Syst 22(10): 1225-1230.

10. Mevorach RA, Hulbert WC, Merguerian PA, Rabinowitz R (1992) Perforation and intravesical erosion of a ventriculoperitoneal shunt in a child with an augmentation cystoplasty. J Urol 147(2): 433-434.

11. Chen T, Lin M, Kung W, Hung K, Chiang Y, et al. (2011) Combined ventriculoperitoneal shunt blockage, viscus perforation and migration into urethra, presenting with repeated urinary tract infection. Ann R Coll Surg Engl 93(7): e151-153.

12. Mihajlović M, Tasić G, Raičević M, Mrdak M, Petrović B, et al. (2012) Asymptomatic Perforation of Large Bowel and Urinary Bladder as a Complication of Ventriculoperitoneal Shunt: Report of Two Cases. Srp Arh Celok Lek 140(3-4): 211-215.

13. Ueda Y, Kakino S, Hashimoto O, Imoto K (1998) Perforation of the bladder by a peritoneal catheter: an unusual late complication of ventriculo-peritoneal shunt. No Shinkei Geka 26(5): 413-416.

14. Yazar U, Kanat A, Akca N, Gazioglu G, Arda IS, et al. (2012) Urethral protrusion of the abdominal catheter of ventriculoperitoneal shunt: Case report of extremely rare complication. J Pediatr Neurosci 7(2): 111-113.

15. De Aguiar GB, Mizrahi C, Aquino JH, Tavares CM, Telles C, et al. (2011) Urethral extrusion of a peritoneal catheter in a patient with a neobladder: a rare complication of shunt insertion. Neuropediatrics 42: 124-127.

16. Yerkes EB, Rink RC, Cain MP, Luerssen TG CA (2001) Shunt infection and malfunction after augmentation cystoplasty. J Urol 165(6 Pt 2): 22622264.

\section{ISSN: 2574-1241}

DOI: $10.26717 / B J S T R .2018 .10 .001942$

Eduardo Moran Pascual. Biomed J Sci \& Tech Res

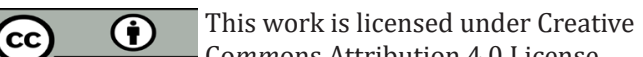

Submission Link: https://biomedres.us/submit-manuscript.php
17. Surchev J, Georgiev K, Enchev Y AR (2002) Extremely rare complications in cerebrospinal fluid shunt operations. J Neurosurg Sci 46(2): 100-103.

18. Barker GM, Läckgren G, Stenberg A, AK (2006) Distal shunt obstruction in children with myelomeningocele after bladder perforation. J Urol 176(4 Pt 2): 1726-1728.

19. Binning MJ, Ragel BT, Walker ML, KJ (2006) Retained peritoneal shunt tubing causing he- maturia. Case illustration. J Neurosurg 104(6 suppl): 434.

20. Pohlman GD, Wilcox DT, Hankinson TC (2011) Erosive bladder perforation as a complication of ventriculoperitoneal shunt with extrusion from the urethral meatus: case report and literature review. Pediatr Neurosurg 47(3): 223-226.

21. De Jong L, Van Der Aa F, De Ridder D VCF (2011) Extrusion of a ventriculoperi- toneal shunt catheter through an appendicovesicostomy. Br J Neurosurg 25(1): 115-116.

22. Kataria R, Sinha VD, Chopra S, Gupta A, Vyas N (2013) Urinary bladder perforation, intra-corporeal knotting, and per-urethral extrusion of ventriculoperitoneal shunt in a single patient: case report and review of literature. Child's Nerv Syst 29(4): 693-697.

23. VL (2001) Methods and complications in surgical cerebrospinal fluid shunting. Neurosurg Clin N Am 12(4): 685-693.

24. Al Fauzi A, Djatisoesanto W, Wahyuhadi J, Parenrengi MA, Turchan A (2017) A Rare Case of Repeated Migration and Transurethral Extrusion of Ventriculoperitoneal Shunt. J Pediatr Neurosci 12(1): 96-98.

25. Hooda R, Khursheed A, Gupta S, Rattan KN (2017) Extrusion of peritoneal end of ventriculoperitoneal shunt through urethra in an infant: A rare complication. Pediatr Urol Case Reports 4: 395-398.

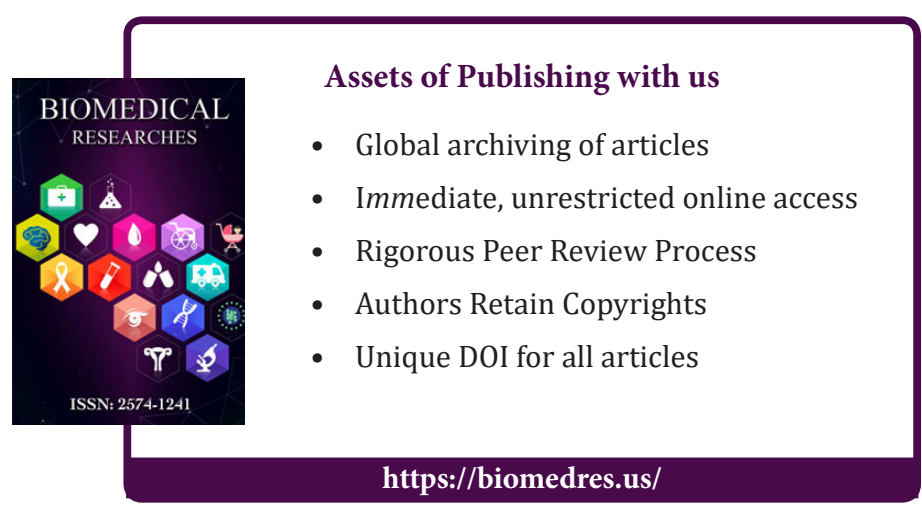

